\title{
Quantum ergodicity and localization in conservative systems: the Wigner Band Random Matrix model
}

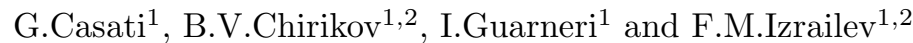 \\ 1 Universitá di Milano, Sede di Como, Via Lucini 3, 22100 Como, Italy \\ ${ }^{2}$ Budker Institute of Nuclear Physics, 630090 Novosibirsk, Russia
}

\begin{abstract}
First theoretical and numerical results on the global structure of the energy shell, the Green function spectra and the eigenfunctions, both localized and ergodic, in a generic conservative quantum system are presented. In case of quantum localization the eigenfunctions are shown to be typically narrow and solid, with centers randomly scattered within the semicircle energy shell while the Green function spectral density (local spectral density of states) is extended over the whole shell, but sparse.
\end{abstract}

PACS number 05.45.+b

One of the main results in the study of the so-called quantum chaos has been the discovery of quantum dynamical localization as a mesoscopic quasi-classical phenomenon [1]. This phenomenon has been widely studied and confirmed by many researchers for dynamical models described by maps. Contrary to a common belief, maps describe not only time-dependent systems, but also conservative ones (in the form of Poincare' maps). On the other hand, to our knowledge, there are no direct studies of quantum dynamical localization in bounded conservative models; moreover, the appearance of dynamical localization in such systems due to quantum effects is challenged by some researchers. The existence of localization in conservative systems would restrict quantum distributions to smaller regions of phase space than classically allowed, and would therefore introduce significant deviations from ergodicity.

We have addressed this problem on the Wigner Band Random Matrix (WBRM) model, which was introduced by Wigner 40 years ago [2] for the description of complex, conservative quantum systems like atomic nuclei. Due to severe mathematical difficulties, the random matrix theory (RMT) immediately turned to the much simpler case of statistically homogeneous (full) matrices, for which impressive theoretical results have been achieved (see, e.g., Refs. [3]). However, full matrices describe local chaotic structures only, and this limitation is often inacceptable, for instance in the case of atoms 4.5 .5 .

Generally speaking, RMT is a statistical theory of systems with discrete energy (and frequency) spectrum. Since the latter is a typical property of quantum dynamical chaos [6], RMT provides a statistical description of quantum chaos and, what is very important, one which does not involve any coupling to a thermal bath, which is a standard element in most statistical theories. Moreover, a single matrix from a given statistical ensemble represents the typical (generic) dynamical system of a given class, characterized by a few matrix parameters. This makes an important bridge between dynamical and statistical description of quantum chaos.

To the extent that Band Random Matrices can be taken as models for generic few-freedoms conservative systems which are classically strongly chaotic (in particular ergodic) on a compact energy surface, the results presented in this Letter provide the first characterization of the properties of quantum chaos in momentum space for quantum systems of this class.

We consider real Hamiltonian matrices of a rather general type (more specific random matrix models have been recently proposed in 7)

$$
H_{m n}=\epsilon_{n} \delta_{m n}+v_{m n} \quad(m, n=1, . ., N)
$$

where off-diagonal matrix elements $v_{m n}=v_{n m}$ are statistically independent, Gaussian random variables, with $\left.<v_{m n}\right\rangle=0$ and $\left\langle v_{m n}^{2}\right\rangle=v^{2}$, if $|m-n| \leq b$, and are zero otherwise. In a classical picture, WBRMs like (11) would correspond to classical Hamiltonians of the form:

$$
H=H_{0}+V
$$

where the perturbation $V$ is usually assumed to be sufficiently small, while the unperturbed Hamiltonian $H_{0}$ is completely integrable. In the quantum model the matrix (11) is given in the basis of the unperturbed eigenstates $\phi_{n}$ of $\hat{H}_{0}$. Correspondingly, the fluctuations of unperturbed energy levels $\epsilon_{n}$ are taken as Poissonian. Although in completely integrable quantum system there is a quantum number for each freedom, we suppose that the unperturbed states are ordered according to increasing energy, and we thereby label them by a single number $n$. The most important characteristic of WBRM is the average level density $\rho$ :

$$
\rho^{-1}=\left\langle\epsilon_{n}-\epsilon_{n-1}\right\rangle
$$

Here and below, the averaging is understood either over disorder (that is, over many random matrices) or within a single, sufficiently large, matrix. Both ways are equivalent owing to assumed independence of matrix elements. 
In the classical case, the unperturbed energy $E_{0}$ is not constant along a classical chaotic trajectory of the full Hamiltonian with a given total energy $H=E$. Instead, it sweeps a range of values, or "energy shell", $\Delta E_{0}=\Delta V$, and is distributed inside this shell according to a measure $W_{E}\left(E_{0}\right)$. The form of $W_{E}\left(E_{0}\right)$ depends on the form of the perturbation $V$; we will call this measure "ergodic" because it is determined by the ergodic (microcanonical) measure on the given energy surface $H=E$. The quantum analog of this measure characterizes the distribution of the "ergodic" eigenfunction (EF) in the unperturbed basis.

Conversely, if we keep the unperturbed energy $E_{0}$ fixed, the bundle of trajectories of the total Hamiltonian $H$, which reach the surface $H_{0}=E_{0}$, has a distribution in the total energy $E$ which is described by a measure $w_{E_{0}}(E)$. In the quantum case, this measure corresponds to the energy spectrum of the Green function (GFS) at energy $E_{0}$, and has received different names, such as "strength function", "local spectral density of states", "spectral measure" of the unperturbed eigenstate at energy $E_{0}$.

An expression for the latter measure has been given by Wigner [2]. For a typical perturbation, represented by a WBRM, the average measure $w(E)=\left\langle w_{E_{0}}(E)\right\rangle$ depends on the Wigner parameter ,

$$
q=\frac{(\rho v)^{2}}{b}
$$

and has the following limiting forms [2] (see also Refs. [9, 10, 11])

$$
w(E)=\left\{\begin{array}{cl}
\frac{2}{\pi E_{s c}^{2}} \sqrt{E_{s c}^{2}-E^{2}}, & |E| \leq E_{s c}, \quad q \gg 1 \\
\frac{\Gamma / 2 \pi}{E^{2}+\Gamma^{2} / 4} \cdot \frac{\pi}{2 \cdot \arctan (1 / \pi q)}, & |E| \leq E_{B W}, q \ll 1
\end{array}\right.
$$

Outside the specified energy intervals, both distributions have exponentially small tails. Formulae (5) are valid provided $\rho v>1$, which is the condition for strong coupling of neighboring unperturbed states by the perturbation. In the opposite case $\rho v<1$ the effect of the perturbation is small, and we have the so-called perturbative localization.

In the limit $q>>1$ we have the semicircle (SC) law and the width of the energy shell $\Delta E=2 E_{s c}=4 v \sqrt{2 b}=$ $4 \sqrt{2 q} E_{b} \gg E_{b}$ where $E_{b}=b / \rho$ is the half width (in energy) of the band. In the other limit, $q<<1$, we have the Breit - Wigner (BW) distribution, of width $\Delta E=2 E_{B W}=2 E_{b}$, with the main part inside a width $\Gamma=2 \pi \rho v^{2}=2 \pi q E_{b} \ll E_{b}$. In all these expressions $E$ is measured with respect to the center of the distribution. Since $q<<1$ requires $\rho v<\sqrt{b}$, in the BW regime the perturbation is not strong enough to couple all states within one bandwidth. This means that the $\mathrm{BW}$ regime corresponds in fact to a sort of partial perturbative localization.

The numerical results presented below are contained in the EF matrix $C_{m n}$, which connects exact eigenfunctions $\psi_{m}$, obtained by diagonalization of the Hamiltonian matrix (1), to unperturbed basis states $\phi_{n}$,

$$
\psi_{m}=\sum_{n} C_{m n} \cdot \phi_{n}
$$

In what follows the eigenvalues $E_{m}$ are ordered, so that $E_{m} \approx m / \rho$.

From the matrix $C_{m n}$ we have found both the statistical distribution $W_{m}(n)=C_{m n}^{2}$ of the eigenstates $\psi_{m}$ on the unperturbed ones $\phi_{n}$, and the distribution $w_{n}(m)$ of the unperturbed eigenstates on the exact ones; the meaning of these distributions is similar to that of the classical $W$ and $w$ discussed above. We have then analyzed both distributions, and have compared their structures to each other and to the SC distribution, paying special attention to localization. By localization we shall here mean a situation, in which eigenfunctions are localized on a scale which is significantly smaller than the maximum one consistent with energy conservation. Indeed, the size of the region which is populated by an eigenfunction (termed localization length in the following) is bounded from above by the ergodic localization length $d^{(e)}=c \rho \Delta E$, which measures the maximum number of basis states coupled by the perturbation. This length characterizes the full width of the energy shell $\Delta E$. The factor $c$ depends on the definition of localization width (see eqn.(7) below). In other words, in a conservative quantum system there is always localization in energy, due to the existence of a finite $\Delta E[8]$. This fact, which is sometimes a source of confusion, is just a trivial consequence of energy conservation. Here we are interested in localization inside the shell [8], which can be caused by quantum effects. In this connection, the matrix size $N$ is an irrelevant parameter, provided $N \gg d^{(e)}$ is large enough to avoid boundary effects. The quantum model (1) is thus defined by the 3 physical parameters $\rho, v$, and $b$.

The localization length $d_{m}$ of a distribution $w_{m}(n)$ can be defined in several ways. We have used the so-called inverse participation ratio (see, e.g., Ref. [6]):

$$
d_{m}^{-1}=\frac{1}{3} \sum_{n} w_{m}^{2}(n)
$$

and similarly for $W_{n}(m)$. The numerical factor $1 / 3$ accounts for fluctuations in individual distributions. These distributions are assumed to be Gaussian and independent [5].

In order to suppress large fluctuations in individual distributions of both types $W_{m}(n)$ and $w_{n}(m)$, we have taken averages over 300 of them, chosen around the center of the spectrum. Since different distributions cover different regions of the $n$ (respectively, $m$ ) space, prior to 
averaging they have to be shifted into a common region. This we have done in two different ways, namely, either by counting the site label $n$ in $W_{m}(n)$ starting from the center of the energy shell i.e., from the reference site $m$ (and vice- versa in the case of $w_{n}(m)$ )(circles in Figs.1,2), or from the center $n_{c}(m)$ of $W_{m}(n)$, defined by

$$
n_{c}(m)=\sum_{n} W_{m}(n) \cdot n
$$

The two types of average will be denoted by $\langle W(n)\rangle, \bar{W}(n)$ respectively. In particular, $\bar{W}(n)$ yields the average shape of an eigenstate (full line in Figs.1,2).

First we shall discuss the distributions $W_{m}(n)$. In \& it was shown that the average localization length $d \equiv$ $\left\langle\left(\sum_{n} W_{m}^{2}(n)\right)^{-1}\right\rangle$ obeys a scaling law of the form

$$
\beta_{d}=\frac{d}{d^{(e)}} \approx 1-\mathrm{e}^{-\lambda}<1
$$

where

$$
\lambda=\frac{a b^{2}}{d^{(e)}}=\frac{a b^{3 / 2}}{4 \sqrt{2} c \rho v}
$$

Here $a \approx 1.2$; factor $c$ can be directly calculated from the limiting expression (5) for $w$, which gives $c \approx 0.92$.

The empirical relation (9) has been found [8] to hold in the whole interval $\lambda \leq 2.5$ and was confirmed in the present studies up to $\lambda \approx 7[12$.

The parameter $\lambda$ has been shown [8] to play the role of an ergodicity parameter because, when it is large, the localization length approaches its maximal value $d^{(e)}$, which means that the eigenfunctions become ergodic, i.e., delocalized over the whole energy shell. Notice that in the $\mathrm{BW}$ region the ergodic localization length $d^{(e)}=\pi \rho \Gamma=2 \pi^{2} b q$, and $\lambda \approx a b / 2 q \pi^{2} \gg 1$ [9] since $q \ll 1$ (and $b \gg 1$ in quasiclassical situations). Hence, localization is only possible in the parameter range in which the local density of states follows the SC law. This domain is the main object of the present studies.

In the case $\lambda \gg>1$ (Fig.1a) we have found that the averaged distributions $\langle W(n)\rangle, \bar{W}(n)$ are fairly close to the SC law: a remarkable result, because that law was theoretically predicted for the other distribution, namely, for the GFS spectrum $\langle w(m)\rangle$. We presume that the deviations from the SC law which are observed in the distribution $\bar{W}(n)$ are due to the not very large value of the ergodicity parameter $(\lambda=3.7)$. The numerical values of the localization parameter (9) are $\beta=0.94$ and $\beta=1.08$ for the two types of average, respectively, in a reasonable agreement with the average $\beta_{d}=0.97$ computed from (9) for $\lambda=3.6$. For finite $q$ the average distributions of both types are bordered by two symmetric steep tails, which apparently fall down even faster than the simple exponential. The structure of these tails will be discussed in detail elsewhere.
The structure of EFs is completely different in the case $\lambda<<1$. (Fig.1b). Whereas individual eigenstates exhibit large fluctuations, the main part of the average distribution ( with respect to the center $n_{c}$ ) $\bar{W}(n)$ shows a clear evidence for exponential localization, with localization length in agreement with the empirical formula (9). The width of the main part is small $(\beta=0.24)$, which is again close to average $\beta_{d}=0.21$ for $\lambda=0.24$. We have found that the main part of the distribution can be represented reasonably well by a simple expression:

$$
\bar{W}(n) \approx \frac{2 / \pi l}{\cosh (2 n / l)}
$$

where the parameter $l$ is related to the localization length by $l=4 \pi^{-2} d$. If, instead of averaging the EFs with respect to their centers, we average them with respect to the center of the energy shell, a nice SC (with some tails) reappears (Fig.1b, $\beta=0.99$ ) in spite of localization . This shows that, in the average, the EFs homogeneously fill up the whole energy shell; in other words, their centers are randomly scattered whithin the shell (see also fig.3). The latter type of averaging provides a new method for calculating ergodic $d^{(e)}$, and hence the important localization parameters $\beta_{d}$ and $\lambda(9)$.

Now we turn to the analysis, in the case $\lambda \ll 1$, of the other type of distribution: the GFS, or local spectral density of states $w_{n}(m)$, which is obtained from the columns of the matrix $C_{m n}$. The structure of this distribution is quite different from that of EFs (represented by matrix rows). Averaging with respect to their centers or with respect to the shell center now yields similar results, which well fit the SC distribution in both cases (Fig.2: $\beta=0.97$ and 0.99 , respectively, cf. Fig.1b with $\beta=0.24$ and 0.99). So, GFS look extended, yet they are localized! This is clear from the average of the corresponding individual $\beta$-values: $\langle\beta\rangle=0.20$. The explanation of this apparent paradox is that, though each GFS is extended over the shell, it is sparse, that is, it contains many 'holes'.

The difference in the structure between EFs and GFS is clear from Fig.3, where solid vertical bars show the main parts of EFs. GFS are represented by horizontal dashed lines whose sparsity immediately follows from scattered localized EFs. Our physical interpretation of the above described structure is the following. Spectral sparsity decreases the level density of the operative EFs (that is, the ones which are actually excited in a given initial state). This is the essential mechanism of quantum localization, via decreasing the relaxation time scale [6] [9]. Yet, the initial diffusion and relaxation are still classical, similar to the ergodic case, which requires extended GFS. On the other hand, EFs are directly related to the steadystate distribution, both being solid because of the homogeneous diffusion during the statistical relaxation.

In conclusion, we have analyzed the structure of the GFS (local spectral density of states) and of the eigen- 
functions for a class of Random Matrices which comes much closer to the structure of the real Hamiltonian matrix of a conservative system than the conventional full Random Matrices. We have provided numerical evidence for the existence of both a delocalized regime, in which eigenfunctions have maximal size, with an average shape close to the semicircle law, and of a localized regime, in which the size of EFs is much smaller than the semicircle width. More precisely, quantum localization introduces a symmetry breaking, in the sense that the eigenfunctions are solid, narrow and randomly scattered inside the energy shell, while the GFS remain extended over the whole shell but become sparse. In classical language, the latter situation means that, although classical trajectories are ergodically distributed over the whole energy surface, the quantum eigenfunctions cover but a small region of the latter. Thus our results indicate that quantum localization is a more general phenomenon than commonly believed, and suggest similar investigations for realistic Hamiltonian, conservative, classically chaotic systems.

Support by the NATO linkage grant LG930333 and by I.N.F.M. is acknowledged. Partial support by grant RB7000 is also gratefully acknowledged by F.M.I.

B.V.C. and F.M.I. are grateful to their colleagues of the University of Milano at Como for their hospitality during the period in which this work was completed.

[1] G.Casati, B.V.Chirikov, J.Ford and F.M.Izrailev, Lect. Notes in Physics,93,(1979), 334 (also in ref.6).

[2] E.Wigner, Ann. Math. 62, 548 (1955); 65, 203 (1957).

[3] T. Brody, J. Flores, J. French, P. Mello, A. Pandey, and S. Wong, Rev. Mod. Phys. 53, 385 (1981); M. Mehta, Random Matrices , (Academic Press, New York,1991).

[4] B.V. Chirikov, Phys. Lett. A 108, 68 (1985).

[5] V.V. Flambaum, A.A. Gribakina, G.F. Gribakin, and M.G. Kozlov, Phys. Rev. A 50, 267 (1994).

[6] G. Casati and B.V. Chirikov, The legacy of chaos in quantum mechanics, in: Quantum Chaos: Between Order and Disorder, edited by G. Casati and B.V. Chirikov (Cambridge University Press, Cambridge, 1995).

[7] V.V.Flambaum, F.M.Izrailev and G.Casati, preprint DYSCO58 Como 1995.

[8] G. Casati, B.V. Chirikov, I. Guarneri and F.M. Izrailev, Phys. Rev. E 48, R1613 (1993).

[9] G. Casati and B.V. Chirikov, Physica D, 86 (1995) 220.

[10] Y.V. Fyodorov, O.A.Chubykalo, F.M.Izrailev, G.Casati, "Wigner random banded matrices with sparse structure: Local spectral density of states", preprint DYSCO 95.

[11] D.M.Leitner and M.Feingold, J.Phys. A 26 (1993) 7367.

[12] the parameter $\lambda$ was introduced to describe energy level statistics in: M. Feingold, D. Leitner, M. Wilkinson, Phys. Rev. Lett. 66, 986 (1991); J. Phys. A 24, 1751 (1991).
FIG. 1. Structure of ergodic (1a) and localized (1b) eigenfunctions. Each figure corresponds to a single matrix with parameters $N=2560, v=0.1, b=16, \rho=40, q=(\rho v)^{2} / b=1$ (a), and $N=2400, v=0.1, b=10, \rho=300, q=90$ (b). The fat full line is the semicircle law (5). Solid lines were obtained by averaging 300 eigenfunctions with respect to their centers; circles, by averaging the same eigenfunctions with respect to the centers of their energy shells. In the ergodic case (a) $\lambda=3.7$, all distributions are close to one another apart from fluctuations. In the localized case (b) $\lambda=0.24$, the average with respect to centers $n_{c}(m)$ of the distributions $W_{m}(n)$ shows a clear localization with $\beta=0.24$, while the other average remains close to semicircle, with $\beta=0.99$.

FIG. 2. Structure of the GFS (local DOS) for a single matrix, with the same parameters as for fig.1b. The same averages as in Fig. 1 are shown, and unlike that case they are close to each other and to the semicircle law.

FIG. 3. A comparison of the structure of eigenfunctions and of GFS in the localized case of fig.1b. Solid vertical bars represent the widths $\Delta n$ of individual eigenfunctions over the unperturbed basis. Horizontal dotted lines show the size $\Delta m$ of the local spectrum for individual basis states. Although all basis states have comparable sizes, close to the size of the energy shell, they are very sparse $(\beta=0.20)$, due to the fact that EFs are strongly localized, and irregularly scattered inside the energy shell. 


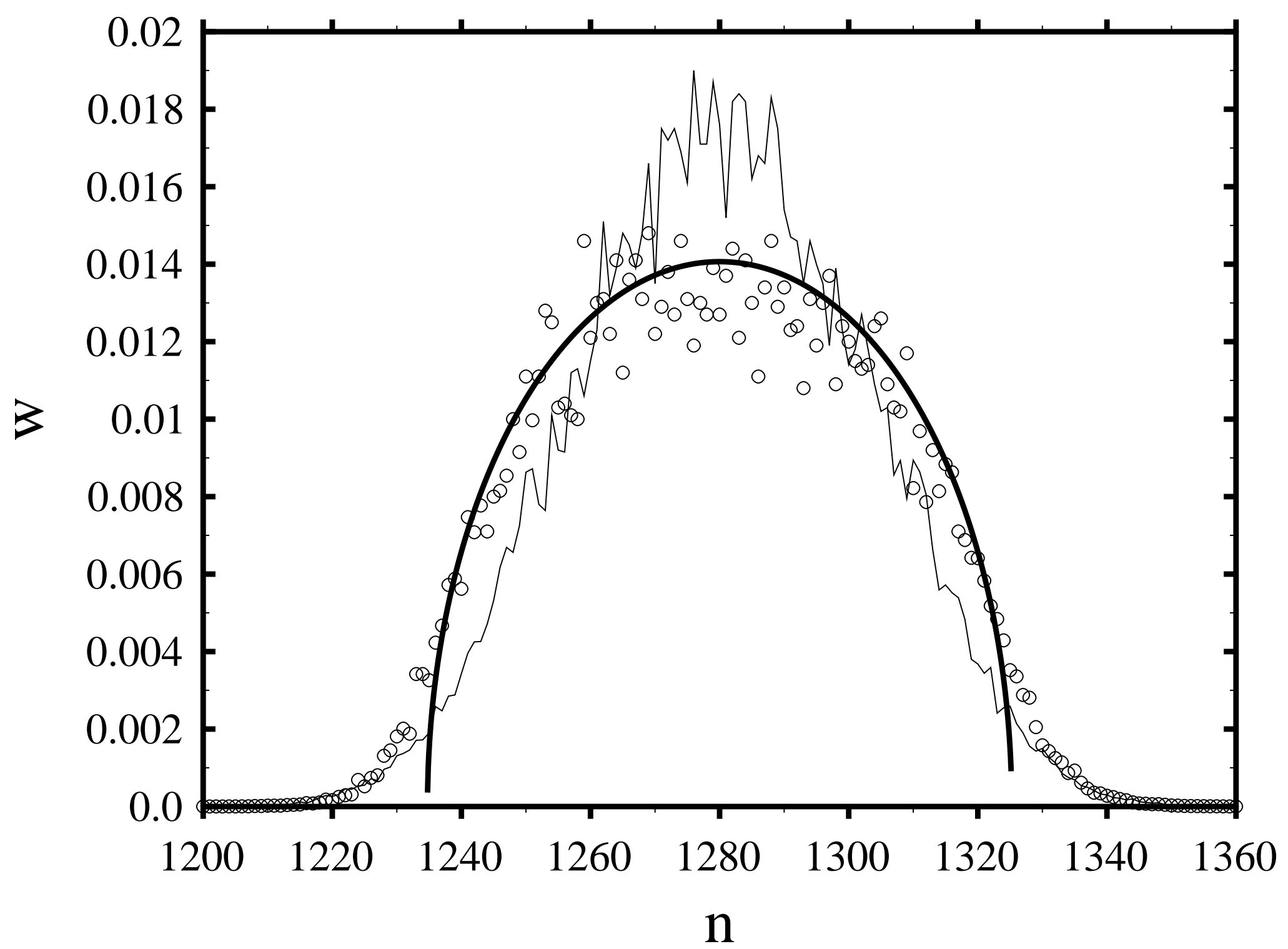




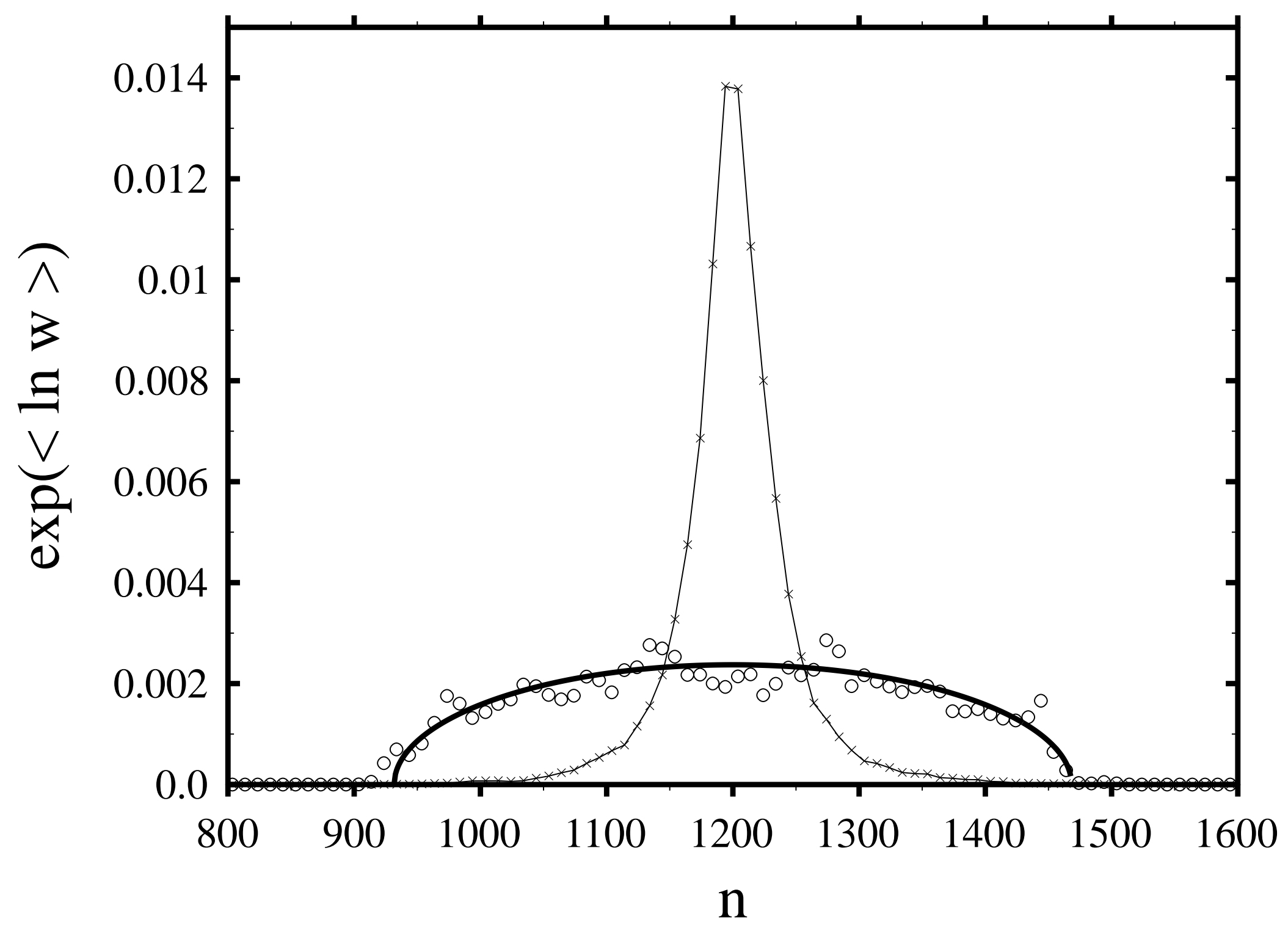




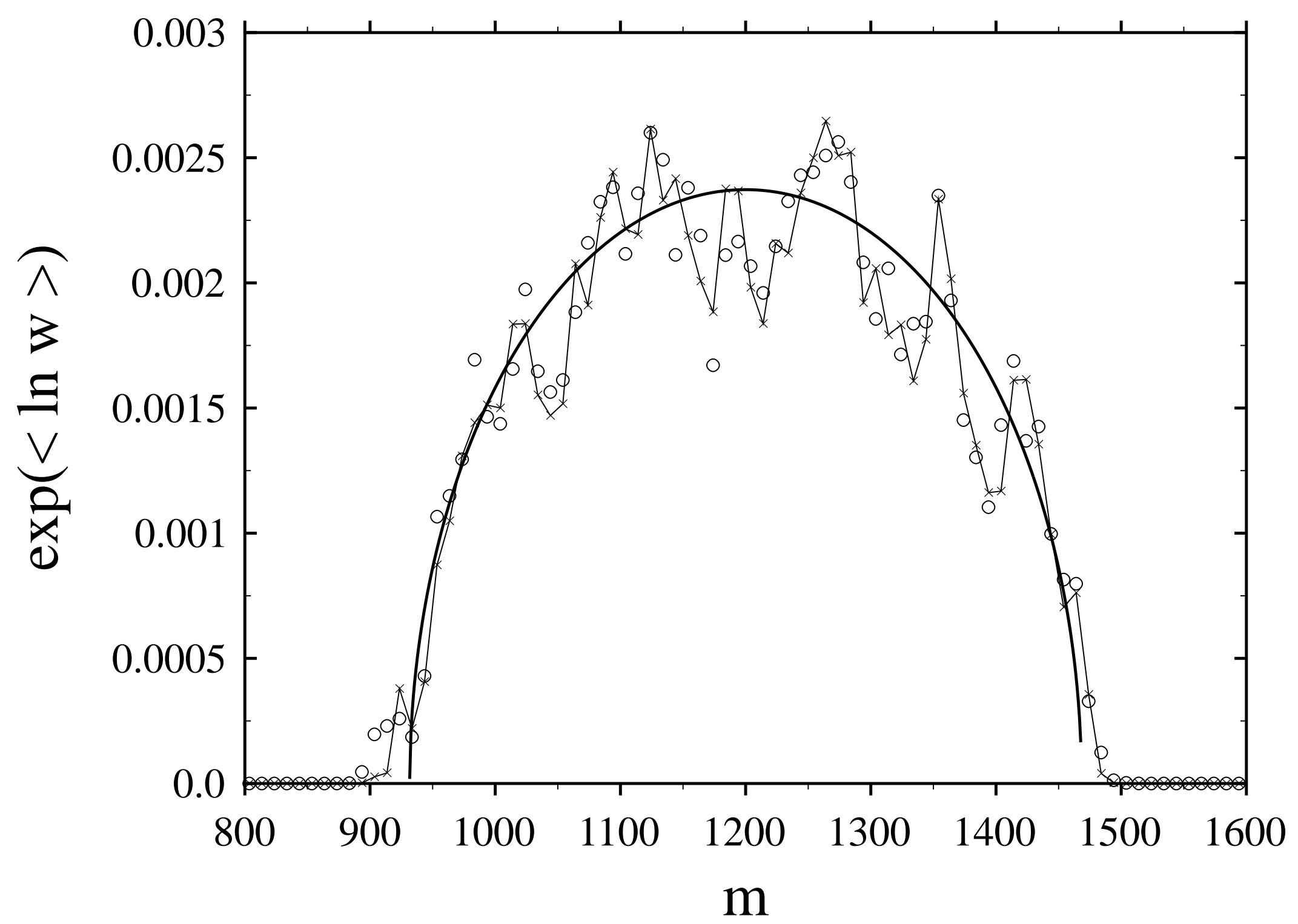




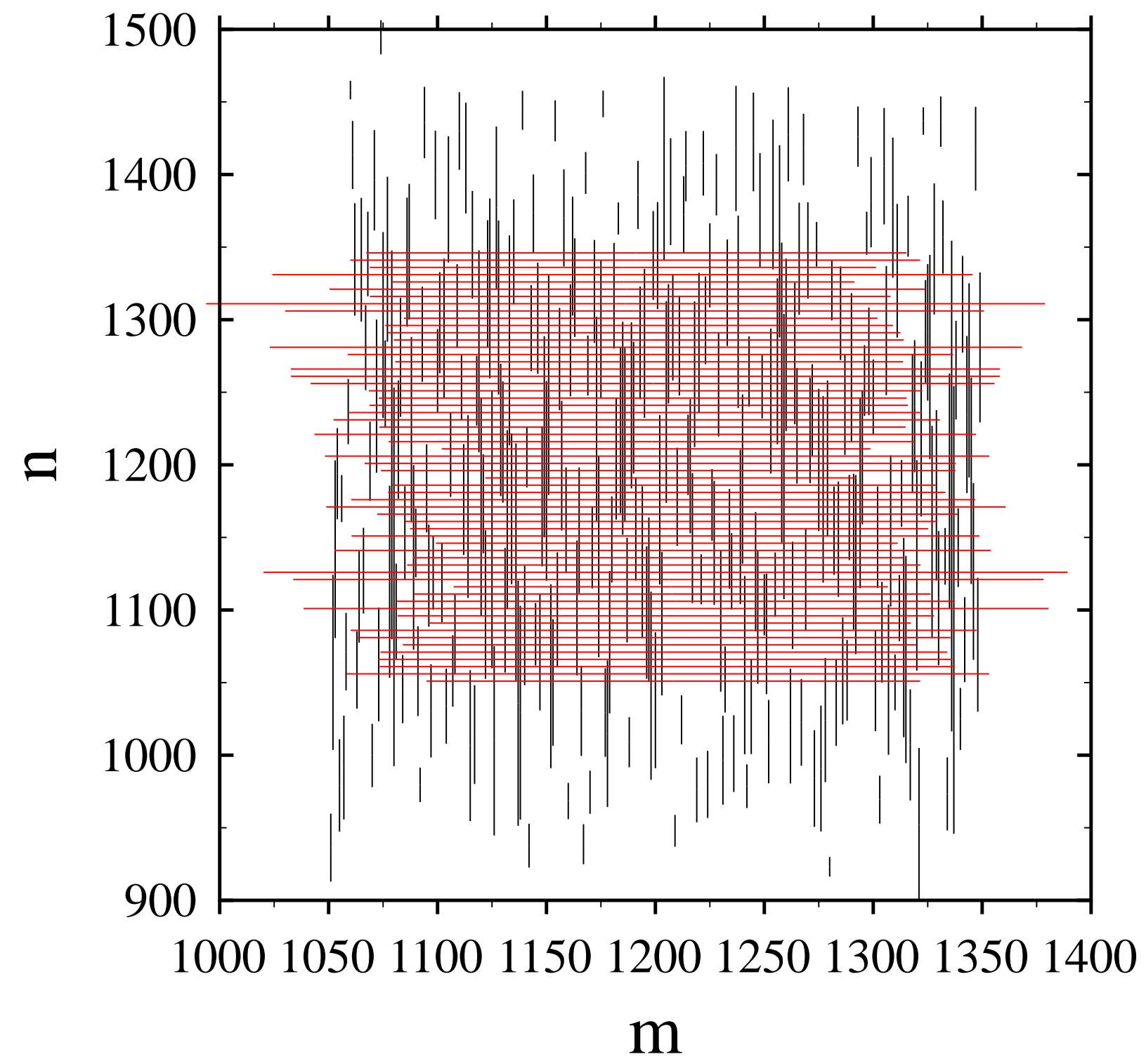

